\title{
Evaluating Arm Movement Imitation
}

\author{
Alexandra Constantin \\ Baxter Hall \\ Williams College \\ Williamstown, Massachusetts 01267 USA \\ Maja J. Matarić \\ Computer Science Department \\ University of Southern California \\ Los Angeles, California 90089 USA
}

Received:January 26, 2006 Accepted: February 16, 2006

\begin{abstract}
In this paper, we present a metric for assessing the quality of arm movement imitation. We develop a joint-rotational-angle-based segmentation and comparison algorithm that rates pairwise similarity of arm movement trajectories on a scale of 1-10. We describe an empirical study designed to validate the algorithm we developed, by comparing it to human evaluation of imitation. The results provide evidence that the evaluation of the automatic metric did not significantly differ from human evaluation.
\end{abstract}

\section{INTRODUCTION}

Imitation, the ability to mimic an observed behavior, appears to be an innate means of acquiring novel skills from observation. In humans, it is vital during development and remains an important aspect of social interaction and adaptation throughout life. From the standpoint of artificial intelligence, imitation can prove to be a fast and efficient form of transferring motor skills between robots or computers and humans.

Our research goal is to gain insight into the mechanisms underlying imitation and to explore the aspects and benefits of humans and machines interacting through the use of imitation. In this paper, we focus on the evaluation of motor imitation, specifically arm movement imitation. As part of our experiments, we gathered arm movement data from people imitating video stimuli, and we developed a metric for evaluating the quality of the imitation. The metric was compared to the evaluation of the same imitation by human observers, in order to gain insight into the efficacy of our method, as well as the mechanisms behind human evaluation of motor imitation.

The rest of this paper is organized as follows: In Section II of this paper we describe the previous research performed in the area of learning movement by imitation. Section III contains a detailed description of our evaluation metric and the components necessary to successfully perform trajectory comparison. In Section IV we describe our experimental design and results. Section $\mathrm{V}$ provides a summary of the paper, as well as a presentation of ongoing and future research.

II.

RELATED WORK

Imitation is a powerful learning mechanism that has raised the interest of both behavioral sciences and robotics. Much research has been done on imitation 
in biological systems and, more recently, there has been a growing interest in imitation in synthetic systems, such as robots and autonomous agents. Here we present only a cursory overview of some activities relevant to the particular project described herein.

With the development of humanoid robots and other articulated systems, motor imitation has become a topic of interest to various robotics research groups: Atkenson and Schaal (1997); Billard and Matarić (1998); ljspeert et al (2001); Jenkins et al. (2000); Matarić (2001) [1-5]. Pomplin and Matarić [6] presented a psychological study of human arm movement imitation, as well as an approach to evaluating imitation which is similar in structure to ours. The evaluation metric did not make a clear enough distinction between pairs of trajectories for the same stimulus and pairs of trajectories of different stimuli. In earlier work the same authors investigated the psychological differences between watching a movement with or without the intention to imitate, as well as features people fixate on while trying to retain a sequence of movement. Their findings were that people watching a movement fixated at the end points, and that the pupils of the subjects watching with the intent to imitate were more dilated than those of the rest of the subjects $[7,8]$.

Other studies by Goncalves et al. [911] compared human movements to the output of a computer vision system, by using a simple mean square error (MSE) metric. They demonstrated that in many cases, even pairs of movements with large MSE were still perceived as identical by human observers. Nehaniv and Dautenhahn [12] have provided a formal framework for the problem of correspondence between dissimilar bodies. Within that framework, Alisandrakis et al. [13] have explored multiple metrics, all of which have been specific to the particular agents and problems being considered. Liu and Geiger [14] and Sebastian et al. [15] presented a framework for silhouette comparison, addressing the issue of identifying topological changes due to the original $3 D$ scenarios and articulations. These methods did not prove to be extendable to $3 D$ movement comparison.

\begin{abstract}
The past research in imitation motivates the research of more sophisticated metrics evaluating the similarity of limb trajectories.
\end{abstract}

III.

\section{APPROACH}

In order to evaluate arm movement imitation, we developed a metric for the distance between arm movement trajectories. Given two trajectories, our metric computes a similarity score on a 1-10 scale. Our aim was to develop a metric yielding higher values for more similar trajectories. The metric comprises three techniques: segmentation, time scaling, and raw trajectory comparison. We describe each in turn in the next section.

Our approach is similar in structure to the work of Pomplin and Matarić [16]. Both their algorithm and ours are based on the techniques of segmentation, time scaling and raw trajectory comparison. The techniques however differ significantly in their design. We decided to apply the evaluation in joint-space, because, unlike Cartesian coordinates, joint angles are independent from the length of subjects' limbs, and thus compensate for physical differences between subjects. We derived six rotational angles from Cartesian coordinates. The angles represented the roll, pitch, and yaw of the upper and lower arms, respectively.

Our evaluation metric compares two arm movement trajectories, $\alpha$ and $\beta$, containing $T_{\alpha}$ and $T_{\beta}$ samples for each of the $\mathrm{J}$ joint angles. The samples will be referred to as $a_{j}(t)$ and $B_{j}(t)$. The metric encompasses three techniques: segmentation, time scaling, and raw trajectory comparison. We describe each in turn next.

\section{a. Segmentation}

The metric heavily relies on segmenting trajectories $\alpha$ and $\beta$ into simpler trajectory-segments $\alpha^{1 \ldots S^{\alpha}}$ and $\beta^{1 \ldots S^{\beta}}$, and comparing each of them individually. The change in direction of joint rotations is a natural indicator of transitions between successive segments in a trajectory. Our approach thus relies on using change in 


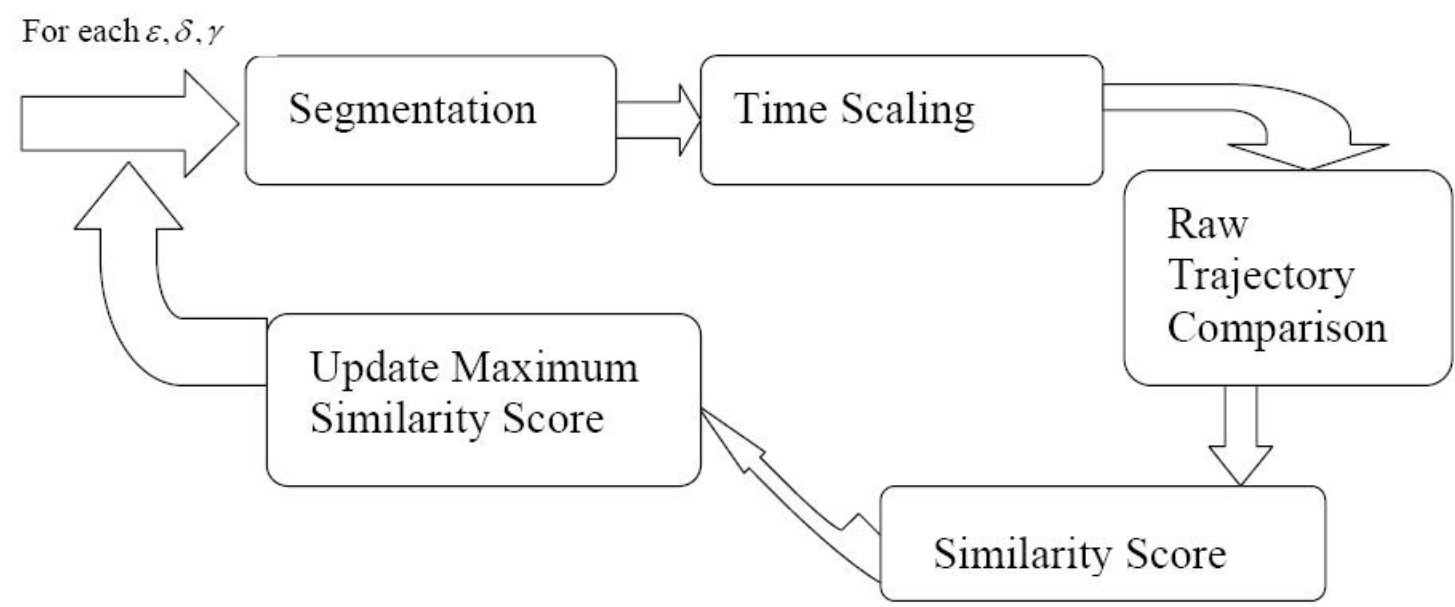

Figure 1. Performance of the Evaluation Algorithm.

direction of joint rotations for segmenting trajectories $\alpha$ and $\beta$.

One challenge of this approach is finding the frame where the direction of joint rotations really changes and is not just the result of system noise. To address this challenge, we used three parameters $-\varepsilon, \delta$ and $\gamma$. $\delta$ represents the minimum difference between two values required to consider the values distinct. $\varepsilon$ represents the minimum number of ensuing frames that need to be consistent with the change in direction of rotation in order for the variation not to be considered noise. A frame $f$ is considered consistent with the change in the direction of rotation joint $j$ if, at any frame $f$, $j$ 's rotation continues to occur in the changed direction within error $\delta . \quad Y$ represents the minimum duration of a segment, in frames.

Instead of estimating appropriate values for $\varepsilon, \delta$ and $\gamma$, the segmentation algorithm performs a parameter space search. It systematically performs segmentations with different combinations of integer values for the three parameters. $\varepsilon$ ranges 1 to $8, \delta$ from 1 to 15 and $y$ from 3 to 8. Empirical investigations found that expanding these intervals does not yield significantly better results. For each combination of segmentations, a distance metric is computed as illustrated in Figure 1, and the segmentation of $\alpha$ and $\beta$ yielding the highest similarity score is used as the final imitation score.

\section{b. Time Scaling}

In order to derive a metric that is invariant to the absolute speed of movement, time scaling is performed for each pair of corresponding trajectorysegments. The shorter of two trajectory segments, say $\alpha^{i}$, is expanded to contain as many samples as its counterpart, $\beta^{i}$. In order to perform this expansion, we noted that the values of rotational joint angles form a continuous function with respect to time. It is impossible for a rotational joint angle to skip from a value $\theta_{1}$ to a value $\theta_{2}$ without taking at least all other values in between.

Formally, let $\hat{\alpha}^{i}$ be the continuous function corresponding to a trajectory segment $\alpha^{i}$. Then, it is reasonable to assume that if $\alpha_{j}{ }^{i}(t)=\theta_{1} \quad$ and $\alpha_{j}{ }^{i}(t+1)=\theta_{2}, \quad$ then $\quad \hat{\alpha}_{j}{ }^{i}(t)=\theta_{1}$, $\hat{\alpha}_{j}{ }^{i}(t+1)=\theta_{2}$, and $\hat{\alpha}_{j}{ }^{i}$ takes all values in between $\theta_{1}$ and $\theta_{2}$ during the time interval $[\mathrm{t} . . . t+1]$. Additionally, because the time interval is very short, it is practical to assume that $\alpha_{j}{ }^{i}$ is monotone on this interval.

$$
\hat{\alpha}_{j}{ }^{i} \text { may thus } \hat{\alpha}_{j}{ }^{i} \text { be approximated }
$$

on each interval $[\mathrm{t} . . \mathrm{t}+1]$ by the following formulas: 


$$
\begin{aligned}
& \hat{\alpha}_{j}^{i}(t)=\theta_{1} \\
& \hat{\alpha}_{j}^{i}(t+1)=\theta_{2} \\
& \hat{\alpha}_{j}^{i}\left(t_{x}\right)=\theta_{1}+\left(t_{x}-t\right) *\left(\theta_{2}-\theta_{1}\right),
\end{aligned}
$$

where $t_{x} \in[t \ldots t+1] . \quad \alpha_{j}{ }^{i}$ is expanded using the $\hat{\alpha}_{j}{ }^{i}$ in the following way:

$$
\alpha_{j}^{i}\left(t_{\text {new }}\right)=\hat{\alpha}_{j}^{i}\left(\frac{t_{\text {new }} * T_{\gamma}}{T_{\omega}}\right) \text {. }
$$

\section{c. Raw Trajectory Comparison}

After identifying and time-scaling two corresponding trajectory segments, $\alpha^{i}$ and $\beta^{i}$, a distance metric is applied in order to determine how similar the two segments are. The distance metric records the number of joint angle samples that were significantly different from each other:

$$
d\left(\alpha^{i}, \beta^{i}\right)=\sum_{t=0}^{T_{\alpha^{i}, \beta^{i}}} \sum_{j=1}^{J}\left|\alpha_{j}^{i}(t)-\beta_{j}^{i}(t)\right|>\varepsilon
$$

Two angles are significantly different from each other if their difference exceeds a threshold, $\varepsilon$. We determined empirically that an appropriate value for the angle-difference threshold, $\varepsilon$, is $25^{\circ}$. Angle differences of $25^{\circ}$ or less are not identified by human observers comparing two movements performed by different persons.

\section{d. Rating the Movement}

After the above three techniques are applied to the movements being compared, an imitation score, $R$, is determined from the percentage of correct joint angle samples across all segments:

$$
R=\min \left(10, \text { round }\left(10 * \frac{\sum_{s=0}^{\min \left(s_{\alpha}, s_{\beta}\right)}\left[d\left(\alpha^{s}, \beta^{s}\right)+\text { Rsamples }\right]}{\text { Nsamples }}\right)+1\right)
$$

where Nsamples is the total number of joint angle samples, and Rsamples is the number of joint angle samples of the trajectory segments with no counterparts (if the movements contain a different number of trajectory-segments).

\section{EVALUATION}

To evaluate the metric we developed, we designed and performed an empirical study which compared the metric to human evaluation of imitation. The study was performed at USC's Interaction Lab, part of the USC Robotics Lab.

\section{a. Experimental Design}

The purpose of this experiment was to test an evaluation metric developed to compare two arm movements. The metric rates the similarity of two arm movements on a scale from 1 to 10 .

\section{i. Participants}

The subject pool consisted of 6 subjects: 1 male and 5 female. Four of the participants were students and two were faculty members. The handedness of the subjects was not taken into account. All subjects were instructed to perform arm movements with the right arm, regardless of arm dominance.

\section{ii. Stimuli}

The stimuli consisted of six video clips, ranging from 4 seconds to 9 seconds in duration. The movements of one of the authors, Alexandra Constantin, were videotaped to be shown as stimuli to the experimental subjects. She participated in a 


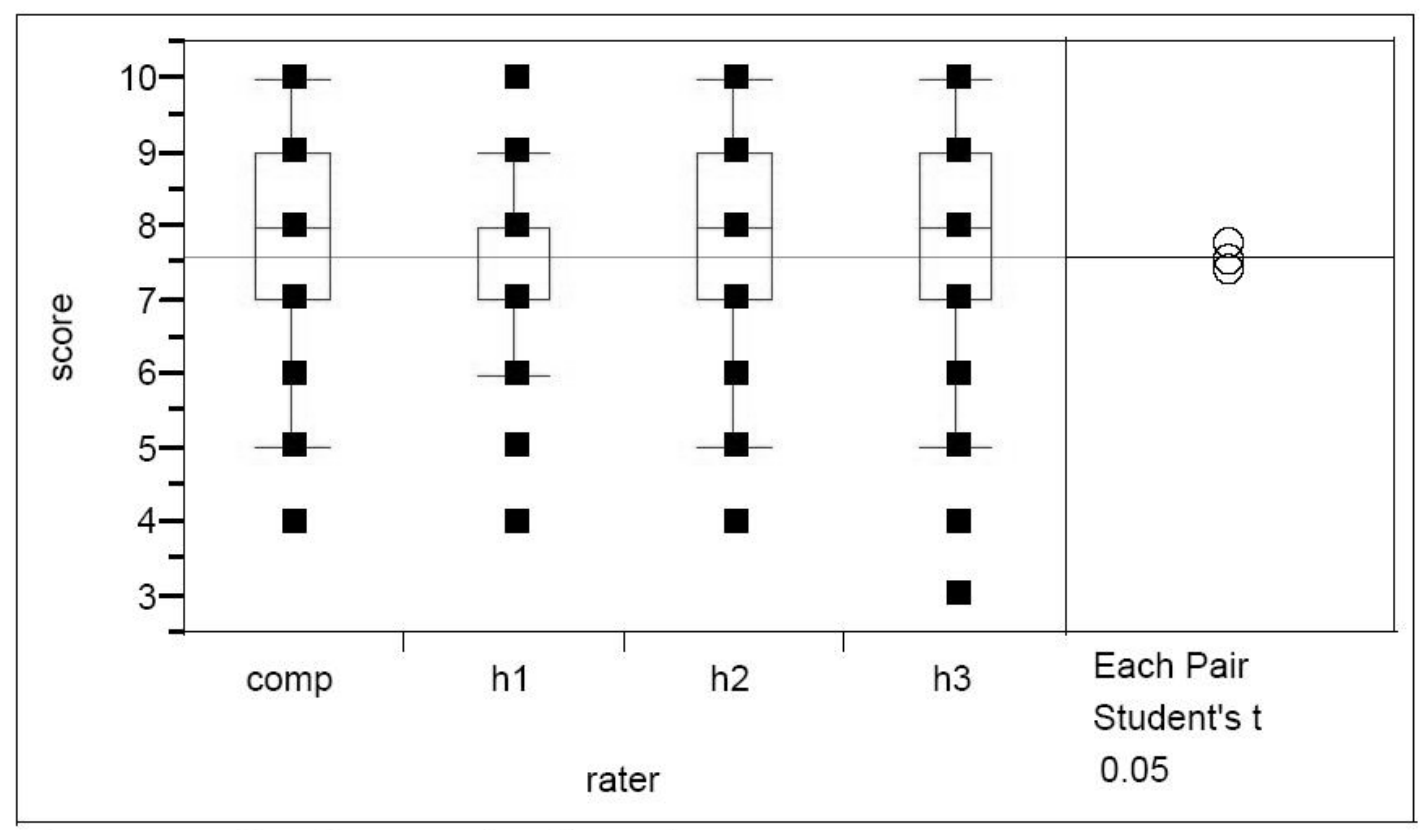

Figure 2. Boxplot of Scores given by Each Rater.

special session to produce reference data for comparison.

Part of the movements contained smooth, circular trajectories. Others were move linear in nature. All movements started with a straightened arm and typically involved movements of the upper arm and lower arm. The stimuli involved no external objects or recognized independent patterns, so that they could be thought of as goal independent; the only goal the subjects had was to imitate the arm movement as accurately as possible.

\section{iii. Apparatus}

Stimuli videos were presented on a 15 inch computer monitor. Subjects' arm movements were tracked using a custommade motion capture system developed in the USC Interaction Lab. The system is comprised of a set of small, lightweight sensors worn on a subject's right arm. Each sensor provides its own global orientation and is physically and computationally independent, requiring only external communication. Orientation information from sensors is communicated via wireless link to a host computer for processing [17].

\section{b. Evaluation of the Data}

i. Method

The subject and the experimenter entered a room equipped with a computer and two motion tracking sensors. The two sensors were strapped on the subject's arm, one above the elbow and one above the wrist. The sensor suit was then turned on by the experimenter, making sure that the sensors were initiated correctly.

Prior to each recording trial, one of the six video stimuli was shown to the subject on the computer monitor. The trials were divided into two conditions: "rehearsal" and "non-rehearsal". In the "rehearsal" condition, the subject was asked to imitate the movement while watching the video. In the "non-rehearsal" condition, the subject was asked to imitate the movement immediately after watching the video.

To initiate the imitation process in either condition, the experimenter gave the verbal signal "ready". The subject then straightened his/her arm. Next, the subject was given the instruction "imitate", at which point $s /$ he started imitating the movement shown in the video (concurrently or immediately following the video). When finished imitating, the subject said "done". 


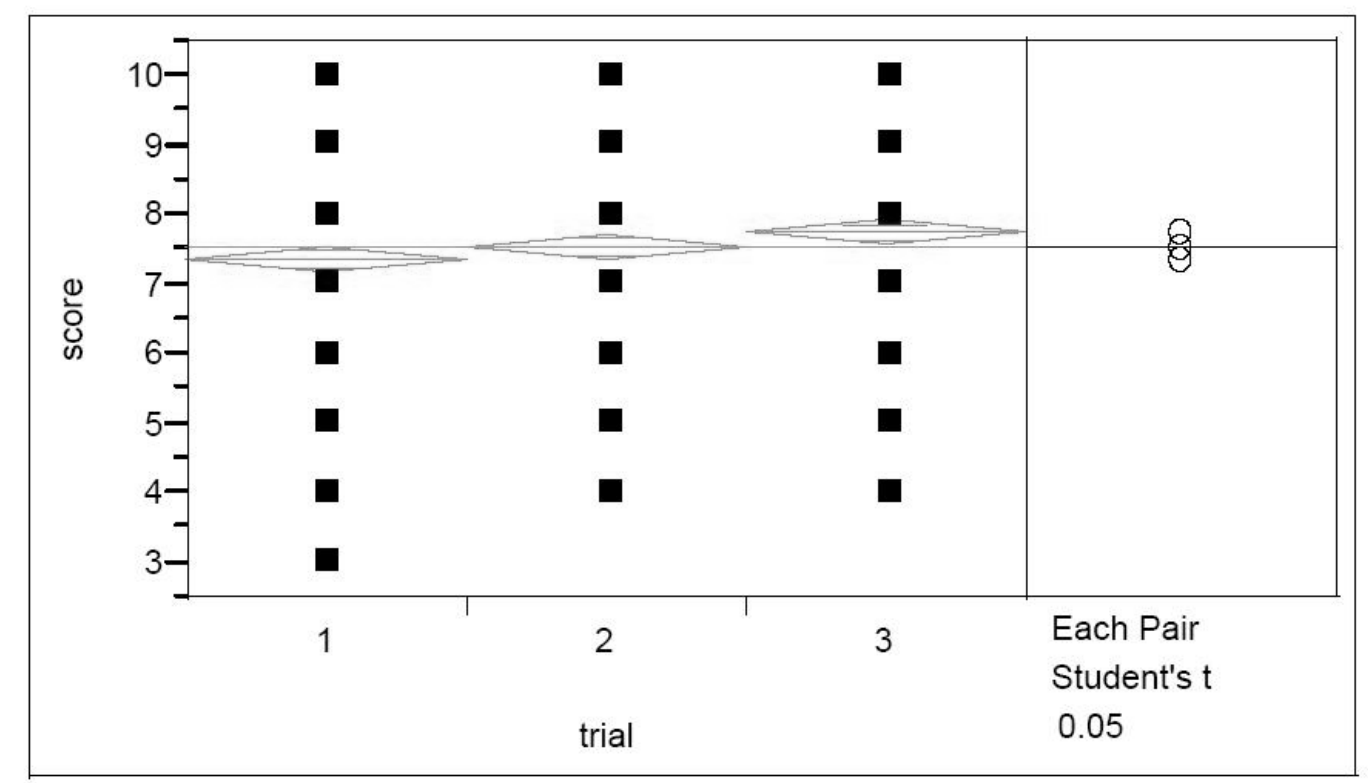

Figure 3. Mean Diamond Plot of Scores Received after Each consequent Imitation (trial).

The movements of the subject were recorded through the motion tracking sensors during the time period between the "imitate" and "done" signals.

Each subject was presented with six stimuli, three in the "rehearsal" condition and three in the "non-rehearsal" condition. The order the stimuli were presented in was randomized across subjects. Each stimulus was shown and imitated three times in succession.

After all movements had been captured, the automated evaluation metric was applied on pairs of files corresponding to the same movement. The performance of the movements was videotaped and observed by three unbiased viewers. The viewers rated the similarity of the movements on a scale of 1-10 for each of the imitation trials, with higher scores corresponding to more similar movements. Finally, the scores of the three evaluators of the same experiments were compared to those computed by the automated metric.

\section{RESULTS AND DISCUSSION}

Our results indicate that, given the variance in human rating, the scores given by the human viewers did not significantly differ from those given by the automated metric. The analysis of variance (ANOVA) test revealed no significant effect of the raters on the imitation scores, $F=1.47, p=$ 0.22. The mean score given by the automated metric was 7.7 ; the mean scores given by the three human viewers were 7.4 , 7.6 and 7.5. A distribution of the scores given by each rater is presented in Figure 2 .

In addition to validating the automated imitation metric, we also acquired additional data about human imitation. Specifically, the subjects' performance significantly improved from the first to the third imitation of the same stimulus, as shown in Figure 3. In addition to this we discovered that when the subject performed the movement while viewing the stimulus, performance did not significantly differ from when imitation was performed after the video had finished.

Given the variance in human ratings, evaluating imitation seems to be very subjective. Human observers varied in their evaluation even when presented with the same pair of movements at different times.

\section{CONCLUSIONS AND FUTURE WORK}

We have described an evaluation metric for human movement imitation. The first results have provided encouraging 
feedback on the use of the metric for evaluating arm movement imitation. Future work could test the accuracy of the metric in evaluating the imitation of more limbs or complete body movements.

Our ongoing work focuses on providing instructive feedback encouraging the learner to work on the least accurate parts of his/her performance with the help of repeated, amplified, and focused demonstrations. Towards this end, we are working on replacing the video stimuli with a 3D skeletal animation.

The successful implementation of this work could lead to an application capable of teaching humans how to perform certain movements, demonstrated either through a graphical interface or by a humanoid robot.

\section{ACKNOWLEDGEMENTS}

The research described here was supported by the CRA-W Distributed Mentoring Program, Summer 2005 Award.

\section{REFERENCES}

1. C.G. Atkeson and S. Schaal. "Robot learning from demonstration" in Proc. $14^{\text {th }}$ International Conference on Machine Learning (Morgan Kaufman Publisher, San Francisco, California, USA, 1997) pp 12-20. URL:citeseer.ist.psu.edu/atkeson97 robot.html.

2. A. Billard and M.J. Matarić. "Learning human arm movement by imitation: Evaluation of biologically inspired connectionist architecture" Robotics and Autonomous Systems, Vol. 35, No. 2-3 (1998) pp 145-160.

3. A.J. ljspeert, J. Nakanishi and S. Schaal. "Trajectory formation for imitation with nonlinear dynamical systems" in IEEE/RSJ/GI International Conference on Intelligent Robots and Systems (IROS 2001) (MIT Press, Cambridge, Massachusetts, USA, 2001) pp 752-757.

4. O.C. Jenkins, M.J. Matarić and S. Weber. "Primitive-based movement classification for humanoid robots" [conference paper] in IEEE International Conference on Humanoid Robots (Humanoid 2000) Cambridge, Massachusetts, USA (October 2000).
5. M.J. Matarić. "Visuo-motor primitives as a basis for learning by imitation: Linking perception to action and biology to robotics" in Imitation in Animals and Artifacts, Kerstin Dautenhahn and Chrystopher L. Nehaniv, Editors. (MIT Press, Cambridge, Massachusetts, USA, 2002) pp. 2865-2870.

6. M. Pomplun and M.J. Matarić. "A segmentation algorithm for the comparison of human limb trajectories," Technical Report (Institute for Robotics and Intelligent Systems, School of Engineering, University of Southern California, Los Angeles, California, USA, 2001).

7. M.J. Matarić and M. Pomplun, "What do people look at when watching human movement?" Technical Report (Brandeis University, Waltham, Massachusetts, USA, 1997).

8. M.J. Matarić and M. Pomplun. "Fixation behavior in observation and imitation of human movement," Cognitive Brain Research, Vol. 7 (1998) pp 191-202.

9. L. Goncalves, E.D. Bernardo, E. Ursella and P. Perona. "Monocular tracking of the human arm in 3D" in Proceedings of the Fifth International Conference on Computer Vision (ICCV 95) (IEEE Computer Society Press, Boston, Massachusetts, USA, 1995) pp 764-770.

10. L. Goncalves, E. di Bernardo and P. Perona. "Reach out and touch space" in Proceedings of the Third International Conference on Face and Gesture Recognition edited by MingHsuan Yang and Narendra Ahuja (Kluwer Academic Publishers, Boston, Massachusetts, USA, 1998) p 234238.

11. Y. Song, L. Goncalves, E. di Bernardo and P. Perona. "Monocular perception of biological motion in Johansson displays" Computer Vision and Image Understanding, Vol. 81, No. 3 (2001) pp 303-327.

12. C.L. Nehaniv and K. Dautenhahn. "Like me? Measures of correspondence and imitation" Cybernetics and Systems, Vol. 32, No. 1-2 (2001) pp 11-51.

13. A. Alisandrakis, C.L. Nehaniv and K. Dautenhahn. "Imitating with Alice: 
Learning to imitate corresponding actions across dissimilar embodiments" IEEE Transactions Systems, Man and Cybernetics-Part A: Systems and Humans, Vol. 32, No. 3 (2002) pp 428-496.

14. Tyng-Luh Liu and Davi Geiger. "Approximate tree matching and shape similarity" Proceedings of the Seventh International Conference on Computer Vision (ICCV '99) (IEEE Computer Society Press, Cambridge, Massachusetts, USA, 1999) pp 456462.

15. T. Sebastian, P. Klein and B. Kimia. "Recognition of shape by editing shock graphs" Proceedings of the Eighth International Conference on Computer Vision (ICCV '01) (IEEE Computer Society Press, Cambridge,
Massachusetts, USA, 2001) pp 756762.

16. M. Pomplun and M.J. Matarić. "Evaluation metrics and results of human arm movement imitation" in Proceedings of the IEEE-RAS International Conference on Humanoid Robots (Humanoids 2000), MIT, Boston, Massachusetts, USA, September 7-8, 2000.

17. N. Miller, O.C. Jenkins, M. Kallmann and M.J. Matarić. "Motion Capture from Inertial Sensing for Untethered Humanoid Teleoperation" in Proceedings, IEEE-RAS International Conference on Humanoid Robotics (Humanoids-2004), Santa Monica, California, USA, November 10-12, 2004. http://rob otics.usc.edu/ maja/publicati

\section{Williams College}

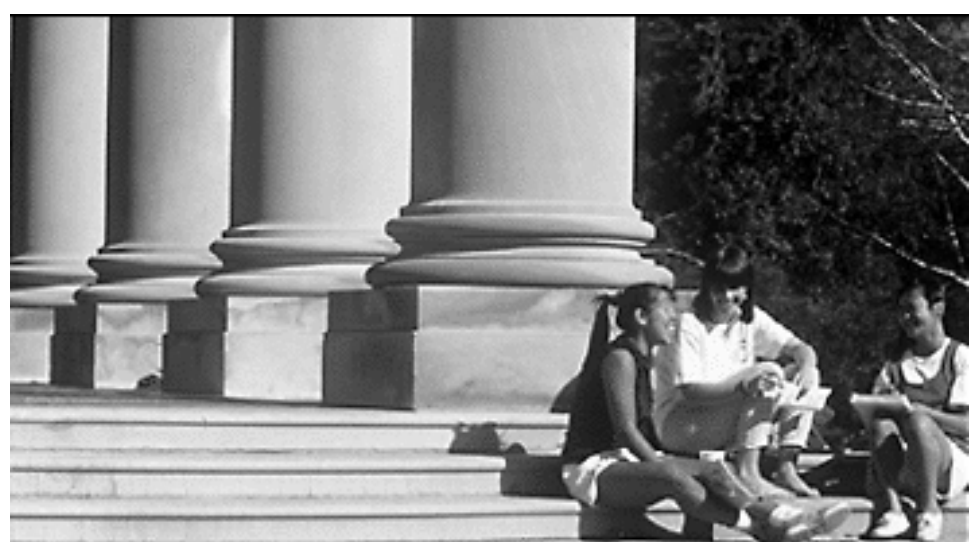

Williamstown Massachusetts, USA www. williams.edu

Established in 1793 with funds bequeathed by Colonel Ephraim Williams, the college is private, residential, and liberal arts, with graduate programs in the history of art and in development economics. The undergraduate enrollment is approximately 2,000 students. Fraternities were phased out beginning in 1962. Coeducation was adopted in 1970.

The school color is purple. The mascot is the Purple Cow. There are three academic divisions (humanities, sciences, social sciences), 24 departments, 33 majors, plus concentrations and special programs. The student: faculty ratio is $8: 1$. The academic year consists of two four-course semesters plus a one-course January term. Williamstown is located in the Berkshires in northwestern Massachusetts, 135 miles from Boston and 165 miles from New York City.

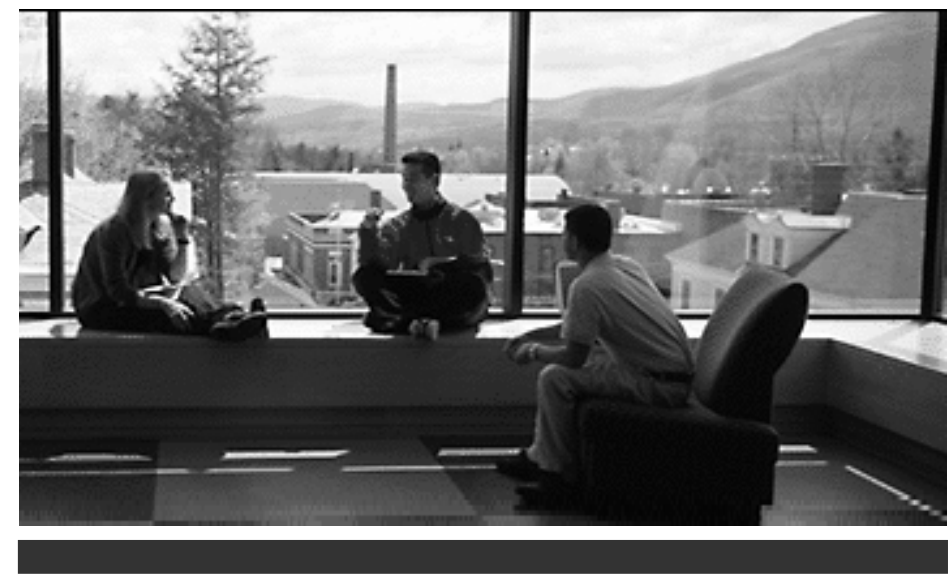

